\title{
¿Sistemas dinámicos en ciencias sociales?
}

\author{
Ignacio Carrasco* \\ Manuel Vivanco**
}

\begin{abstract}
Resumen
Los sistemas dinámicos son uno más de los distintos tipos de sistemas complejos, a saber: sistemas autorreferenciales, sistemas autopoiéticos, sistemas disipativos, sistemas emergentes, sistemas complejos adaptativos, etc. En el contexto de la modelación matemática en ciencias sociales, en el presente artículo se introduce una definición básica de sistemas dinámicos, así como sus principales conceptos. Luego se describen antecedentes clave en cuanto a las aplicaciones ya realizadas en ciencias sociales y se profundiza en una breve aplicación en el ámbito de la economía de la pesca artesanal en que es relevante el uso de sistemas dinámicos. Finalmente se plantea una discusión sobre los desafíos de la investigación con sistemas dinámicos en ciencias sociales.
\end{abstract}

Palabras clave: Sistemas dinámicos - teoría del caos - no linealidad - modelamiento.

\begin{abstract}
Dynamical systems are a specific kind of complex systems, namely: self-reference systems, autopoietic systems, disipative systems, adaptative complex systems, etc. In the context of mathematical modelling in social sciences, this article introduces a basic definition of dynamical systems and its main concepts. A background about key applications in social sciences is shown, and a specific case of the artisanal fisheries in which dynamical systems are certainly useful. Finally, some of the current research challenges of dynamical systems in social sciences are discussed.
\end{abstract}

Keywords: Dynamical systems - chaos theory - non-linearity - modelling.

* Sociólogo, Universidad de Chile. Magíster en Gestión de la Inmigración, Universidad Pompeu Fabra, Barcelona, España.

** Doctor en Sociología, Universidad Complutense de Madrid, España. Docente del Departamento de Sociología de la Facultad de Ciencias Sociales de la Universidad de Chile. 


\section{INTRODUCCIÓN}

La claridad sobre qué son, para qué sirven, y cómo puede ser una investigación en que el uso de sistemas dinámicos sea pertinente, es escasa dentro del campo de las ciencias sociales en Chile. A menudo se confunde con la dinámica de sistemas desarrollada por Jay Forrester, con la técnica de análisis de series temporales, o se incluye como parte de la teoría general de sistemas de Luhmann. Lo cierto es que los sistemas dinámicos se enmarcan en una teoría matemática cuyos conceptos y técnicas se aplican a un amplio espectro de fenómenos (Capra, 1998) y que por lo tanto pueden clasificarse en el contexto de la Teoría de la Complejidad (Vivanco, 2010). La complejidad no estudia sistemas que son ordenados y predecibles -como el movimiento de los cuerpos celestes- ni irreductiblemente azarosos -como la propagación del incendio en una pradera-. Los sistemas ordenados se caracterizan porque sus componentes siguen una legalidad que evidencia cómo cada componente depende de otro. Los sistemas desordenados -que no son azar irreductible- si bien no pueden ser descifrados considerando el comportamiento de cada uno de sus componentes sí lo son respecto al comportamiento del promedio de éstos. La complejidad no es la mecánica y tampoco la estadística. Su ámbito de competencia es un terreno intermedio que los miembros del Instituto de Santa Fe denominan "al filo del caos". Langton (1990) y Kauffman (2003) cultivan esta metáfora que es título de un libro: Complejidad. Una ciencia emergente al filo del orden y el caos (Waldorp, 1992).

En este contexto, la pertinencia que los sistemas dinámicos tienen en las ciencias sociales está dada a partir de dos conceptos matemáticos que vienen a ser parte de la ciencia de la complejidad, no linealidad y temporalidad. En este sentido es que es posible identificar un amplio rango de aplicaciones que van desde aproximaciones metafóricas y semánticas hasta intentos por establecer una continuidad con la teoría de sistemas dinámicos. De hecho, en el periodo 1980-1995 las ciencias sociales (en particular en Francia) consideraron a la teoría del caos como parte de su reflexión. Por ejemplo, Castoriadis (1993) discute algunas consecuencias epistemológicas de la definición de caos y Chatelet (1995) critica el uso de atractores caóticos en economía. Sin embargo, sin una comprensión rigurosa con las definiciones básicas de sistemas dinámicos, conceptos como caos y atractor (entre otros) se diluyen en una pirotecnia conceptual cuyo desempeño teórico-práctico es a lo menos escuálido.

Con el objetivo de aportar al sentido de los sistemas dinámicos en ciencias sociales, en este artículo interesa responder a tres cuestiones. En primer lugar, introducir una definición básica de sistemas dinámicos, así como sus principales conceptos, tales como atractor, caos y espacio de fases. En segundo lugar, introducir antecedentes en cuanto a las aplicaciones ya realizadas en ciencias sociales, profundizando en algunos de ellos. En tercer lugar, se presenta una breve aplicación en el ámbito de la economía de la pesca artesanal en que es relevante el uso de sistemas dinámicos, ya que se trata de un problema esencialmente dinámico y no lineal, la sostenibilidad de la pesquería de merluza común en la quinta región de Chile. Por último, se plantea una discusión sobre los desafíos de la investigación con sistemas dinámicos en ciencias sociales. 


\section{DEFINICIONES BÁSICAS DE SISTEMAS DINÁMICOS}

Un sistema dinámico continuo es en términos formales una función continua $\varphi: R \times$ $X \rightarrow X$ (donde $R$ el conjunto de los números reales) tal que $\varphi(0, x)=x$ y $\varphi(t, \varphi(s, x))=\varphi$ $(t+s, x)$. En términos coloquiales, pueden entenderse como una o más funciones cuyo comportamiento puede conocerse de acuerdo a ciertos parámetros, variables e interacciones entre variables. Es importante destacar que $X$ corresponde a lo que más adelante se define como espacio de fases ${ }^{1}$.

En rigor, en este artículo solo se tratan dos tipos particulares pero muy notables de sistemas dinámicos, a saber: las ecuaciones en diferencias ${ }^{2}$ (ED) (sistema dinámico discreto) y las ecuaciones diferenciales ordinarias (EDO) (sistema dinámico continuo). Sin embargo, dadas las diversas alusiones a la teoría del caos desde las ciencias sociales, es pertinente referirse brevemente a algunos de sus conceptos. Específicamente, la teoría del caos estudia aquellos sistemas dinámicos que tienen un comportamiento caótico. Es interesante mencionar un listado somero de dicotomías puestas en agenda:

- Técnica de análisis de datos versus nuevo enfoque epistemológico.

- Coordenadas espacio temporales estándar o nueva definición del espacio de fases.

- Tiempo reversible de la física o tiempo irreversible de la evolución.

- Azar versus determinismo.

- Orden degradado en desorden versus desorden generador de orden.

- Linealidad o no linealidad.

- Proporción causa-efecto o desproporción causa-efecto.

- Geometría con dimensión racional (fractal) o geometría con dimensión natural (no fractal).

La teoría del caos disuelve algunas de las oposiciones expuestas. A saber, azar y determinismo, orden y desorden, reversibilidad e irreversibilidad del tiempo. Actualiza viejos conceptos como no linealidad y evidencia nociones nuevas como bifurcación o efecto mariposa. ¡El libro en que muchos nos iniciamos (Gleick ${ }^{3}$, 1988) afirma que la teoría del caos es una revolución sólo comparable a la teoría de la relatividad o la mecánica cuántica! Sistemas que evolucionan según un patrón de comportamiento identificado y que a veces son impredecibles a largo plazo.

La teoría del caos se enmarca en el estudio de los sistemas dinámicos no lineales. La noción de no linealidad hace referencia a la no proporcionalidad entre la causa y el efecto, que en definitiva dependen de las variables que se estén incorporando en un modelo o ecuación determinada. En el origen de la no proporcionalidad está la sensibilidad a las condiciones

1 Además, $X$ puede ser de dimensión infinita, por ejemplo, $X=C([0,1], R)$, el espacio de las funciones continuas de $[0,1]$ que toman valores en $R$.

2 Algunas referencias sobre este tipo de ecuaciones son Saber (1991) y Walter y Peterson (2000).

3 Aunque ciertamente es importante recordar que dicho libro es una obra de divulgación científica escrita por un periodista del New York Times. 
iniciales. Formalmente implica que pequeñas perturbaciones en la evolución de una órbita genera otra órbita que se inicia en un punto cercano a la primera y se desarrolla amplificando la diferencia respecto a la órbita inicial. Devaney (2003) plantea una definición que, si bien no es intuitiva, debe tenerse en cuenta. Sea $V$ un conjunto, $f: V \rightarrow V$ es caótica en $V$ si:

- $f$ tiene sensitividad a las condiciones iniciales.

- $f$ es topológicamente transitiva.

- Los puntos periódicos son densos en $V$.

Los sistemas dinámicos que presentan un comportamiento caótico son no lineales y aperiódicos. Se produce un comportamiento aperiódico cuando ninguna variable que describe el estado del sistema da lugar a una repetición regular de valores. En consecuencia, el comportamiento del sistema no produce una regularidad empírica evidente. Un comportamiento aperiódico -sin periodos- genera para ciertos $\operatorname{casos}^{4}$ un conjunto de medidas azarosas que no permiten realizar predicciones exactas. Es importante mencionar que desde la teoría del control se han propuesto herramientas para el control de sistemas caóticos, tales como las planteadas por Ditto y Percora (1993).

Paradójicamente, son sistemas aperiódicos y deterministas. Uno de los atributos epistemológicamente más relevantes de la teoría del caos es que estudia sistemas aleatorios y, simultáneamente, deterministas. Desde el punto de vista formal el sistema es determinista. A saber, el modelo matemático expresa la ley que rige la evolución del sistema. El comportamiento del sistema es caótico porque siendo determinista es impredecible.

\subsection{Las preguntas del caos}

Las respuestas de la teoría del caos no son a preguntas del tipo ¿por qué? Responde a interrogantes referidas al modo en que funcionan los sistemas dinámicos no lineales en ciertas condiciones. ¿Cómo se puede conocer el patrón de comportamiento en fenómenos altamente aleatorios que son fundamentalmente impredecibles? ¿Cómo el orden subyacente persiste en un sistema que evoluciona desordenadamente? Berge et al. (1994) plantean la disyuntiva de la teoría del caos. "La meta final es comprender el origen y las características de la evolución en el tiempo de sistemas que presentan niveles de desorganización que los hace ininteligibles".

\subsection{Determinismo}

Un sistema determinista o aleatorio o caótico es esencialmente distinto. El sistema determinista se caracteriza porque a un suceso le sucede sólo un resultado posible. En consecuencia, conocidas las condiciones iniciales el sistema evoluciona según leyes que rigen el proceso.

4 Es interesante notar el caso de funciones casiperiódicas que tienen un comportamiento aperiódico, como por ejemplo $f(x)=\sin (t)+\cos (\pi t)$. En algunos trabajos se habla de pseudoaleatoriedad (tales como Besicovitch, 1952). 
En un sistema aleatorio la secuencia de sucesos es independiente entre sí. El orden de los sucesos es regido por el azar. En una secuencia de sucesos aleatorios a un suceso dado le puede suceder otro cualquiera de la serie sin ninguna restricción.

Un sistema caótico es aleatorio y determinista. El suceso antecedente puede generar un resultado impredecible. Sin embargo, el sistema en su conjunto responde a un patrón determinista. La teoría del caos es contraintuitiva como la teoría de la relatividad y la mecánica cuántica, en el sentido de que dos puntos muy cercanos pueden seguir trayectorias completamente diferentes. La intuición es un instrumento feble. Nada más contraintuitivo que la luz se curve o que una partícula sea onda y corpúsculo. La teoría del caos enfrenta la intuición no sólo a propósito de que cambios pequeños pueden producir consecuencias gigantes. Más contraintuitivo es estudiar sistemas que son simultáneamente deterministas y aleatorios. Sistemas donde el orden emerge del desorden y viceversa.

\subsection{Espacio fase}

El espacio fase es el lugar geométrico en que se representa la trayectoria del sistema dinámico. Permite que la evolución del sistema sea descrita exhaustivamente.

El espacio de fase es un espacio abstracto constituido por todos los posibles valores de las $n$ variables de estado que componen el sistema. Así, por ejemplo, para dos variables el espacio de fase es un plano; tres variables constituyen un espacio tridimensional y sucesivamente para espacios $n$ dimensionales.

Conocidos los valores de las variables de estado de un sistema dinámico éste se puede proyectar en cada uno de los $n$ ejes del espacio fase. Nótese que la evolución del sistema se explica por la evolución en conjunto de las variables. La ley dinámica del sistema que define su evolución también define la evolución en el tiempo de cada una de las $n$ variables que componen el espacio fase. El espacio de fases es un hipotético espacio multidimensional en el que se representa la evolución de un sistema dinámico. Cada punto del espacio fase representa un momento en la evolución del sistema. Las coordenadas del punto corresponden a los valores que asumen las variables para un determinado momento del sistema. La evolución del sistema se representa por las órbitas que son la huella dejada en el espacio de fases por las diversas variables.

\subsection{Atractor extraño}

Existen sistemas dinámicos no lineales que presentan atractores no extraños. En términos matemáticos, los atractores son básicamente puntos en el espacio fase que atraen la mayor parte de las soluciones o trayectorias del sistema.

La noción de atractor extraño fue elaborada por David Ruelle y Floris Takens (1971) para describir el vórtice o remolino generado por un líquido que fluye con turbulencia. Geométricamente un atractor extraño es un subconjunto de puntos en el espacio de fase. Los puntos conforman un objeto ajeno a la comprensión de la geometría euclídea. En efecto, 
la dimensión del atractor extraño no es un número entero. No es una recta de dimensión 1, tampoco un plano de dimensión 2, ni un volumen de dimensión 3. Un atractor extraño es un objeto fractal (Grassberger y Procaccia, 1983).

El atractor extraño es generado por repetidas iteraciones de un sistema determinista que evoluciona caóticamente. Corresponde a una región del espacio fase que captura las órbitas atrayéndolas a un punto atractor.

El atractor evidencia la presencia de orden en el caos. El orden queda al descubierto examinando el lugar geométrico que representa al sistema. El atractor extraño es la ilustración gráfica de la evolución de éste. Evidencia el carácter determinista del sistema y enseña su historia. Geométricamente el atractor extraño es una curva de longitud infinita y se extiende en un espacio finito sin orbitar dos veces en el mismo lugar.

El atractor de Lorenz es mítico porque es un descubrimiento casual que marca el inicio de la ciencia del caos. Lorenz desarrolló un sencillo modelo de doce variables y tres ecuaciones para estudiar el comportamiento climático. Sin percatarse cambió de tres a seis el número de decimales en los cálculos del computador. Trabajando con seis decimales sorprendentemente la gráfica cambió. Se dibujó el primer atractor extraño. Las mismas ecuaciones deterministas cambiando mínimamente las condiciones iniciales se comportaban de modo caótico. El gráfico se presenta a continuación (Figura 1) desplegándose como una mariposa en que se reitera el patrón de comportamiento del sistema. Gráficamente el atractor extraño es una curva infinita domiciliada en un espacio con perímetro finito.

\subsection{La ecuación logística}

Una manera de explicar los conceptos desarrollados anteriormente es presentar uno de los sistemas dinámicos más populares, la ecuación logística, que a pesar de tener un comportamiento determinista en su versión continua, es ciertamente ilustrativa. En este

FIGURA 1

Soluciones de la ecuación Lorenz

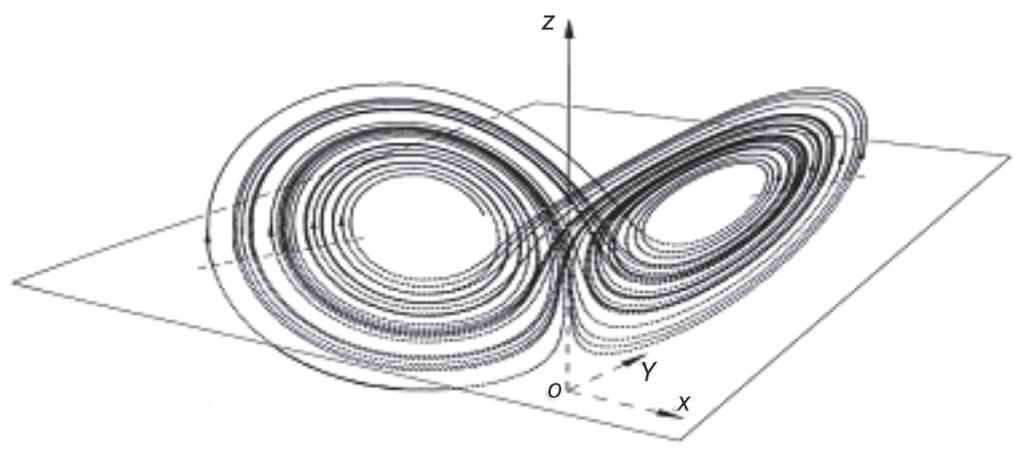

Fuente: Waldorp (1992). 
ejemplo interesa por un lado poner en práctica algunas de las definiciones desarrolladas en relación con la teoría de sistemas dinámicos y comportamiento caótico.

El mapa logístico es una ecuación diferencial o en diferencia (dependiendo de la definición discreta o continua del tiempo) desarrollada por el matemático belga Pierre François Verhulst en 1838. En el siglo XX, diversos científicos vinculados principalmente al campo de la biología han trabajado sobre esta ecuación (Thieme, 2003; Murray, 1989). Con ésta es posible modelar el crecimiento poblacional, asumiendo que los estados iniciales de desarrollo serán exponenciales, pero a medida que el tamaño de la población aumenta, la tasa de reproducción disminuirá. En este sentido, en el modelo se plantea la lógica del crecimiento autolimitado en tanto la tasa de reproducción es proporcional a la población existente y la cantidad de recursos disponibles. Específicamente, la familia de sistemas dinámicos asociados a la ecuación logística tiene la siguiente forma:

$$
F \mu=\mu x(1-x)
$$

Donde $x$ se encuentra entre $[0,1]$ y el parámetro $\mu$ toma valores en el intervalo $[0,4]$. Cuando el parámetro $\mu$ se encuentra entre los valores 1 y 3 se presenta un comportamiento de equilibrio caracterizado por la convergencia de las órbitas a puntos fijos. Sin embargo, al observar el espacio de fases de las órbitas de la ecuación logística se aprecia un cambio en la estructura de los puntos fijos, generado principalmente por divisiones de las trayectorias o lo que se define también como bifurcaciones. Si bien para los valores de $\mu$ entre 3 y 4 permanecen los puntos fijos, aparecen puntos periódicos de periodo 2, producidos por un tipo de bifurcación conocido como duplicación de periodo. Esta duplicación va acompañada de algo muy novedoso, ya que al pasar el valor 3,449 los puntos periódicos de periodo 2 se convierten en repulsivos y se duplican, cada uno de ellos, para dar una órbita periódica de periodo 4 que será atractiva hasta aproximadamente $\mu=3,55$ (Martín et al., 1998).

Aquí se está en presencia de lo que se llama duplicación de periodo como ruta al caos, en tanto si se continúa observando el sistema dinámico, aparecerán múltiples bifurcaciones que seguirán duplicando los puntos periódicos. Al ir avanzando hacia un valor del parámetro $\mu=4$ se va reproduciendo el fenómeno anterior, y sucesivamente van apareciendo órbitas atractivas y repulsivas. La Figura 2 muestra el espacio de fases en que se representa cómo las órbitas se bifurcan, cambiando la estructura de los puntos fijos. Una primera duplicación de periodo ocurre con la bifurcación generada luego de que $\mu$ es superior a 3. Luego, también se aprecia una duplicación de periodo 4. Nótese cómo este fenómeno va ocurriendo cada vez en rangos más cortos de $\mu$, siendo finalmente imposible distinguir gráficamente de cuántas bifurcaciones se trata.

Finalmente, la iteración de la ecuación logística para valores del parámetro $\mu=4$ genera un atractor extraño y un comportamiento caótico de las órbitas del sistema. En la Figura 3 se presenta el gráfico generado después de 10, 50 y 100 iteraciones. La Figura ilustra la evolución de un sistema que para ciertos valores del parámetro se comporta según un orden caótico. Nótese que no es posible predecir la trayectoria exacta de la próxima órbita. Sin embargo, es fácil señalar que ocupará un espacio que reforzará la figura que forma el atractor extraño. 
FIGURA 2

Diagrama de Feigenbaum

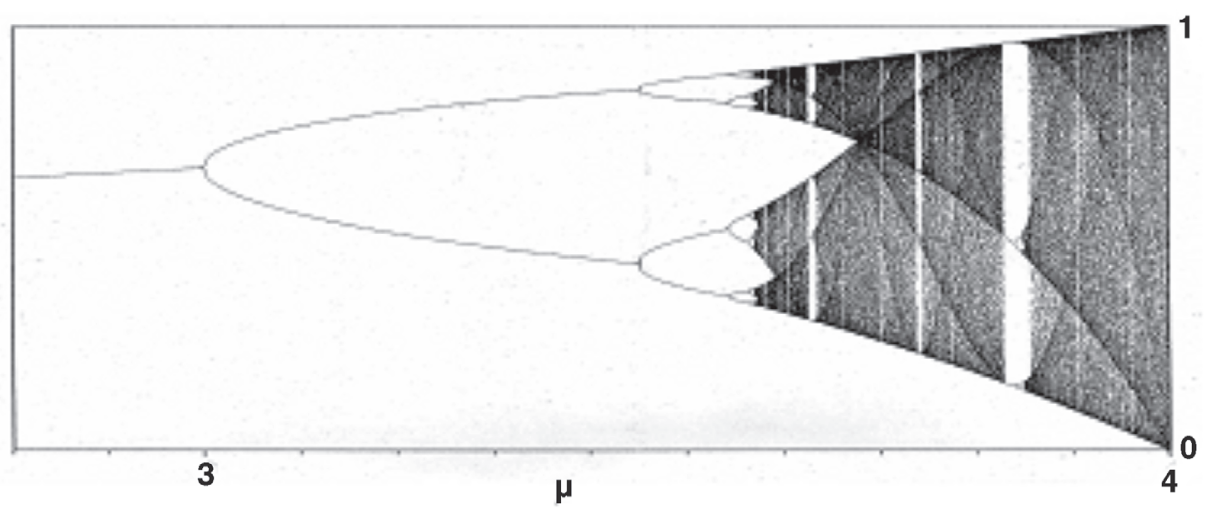

Fuente: Martín et al. (1998).

FIGURA 3

Iteraciones ecuación logística
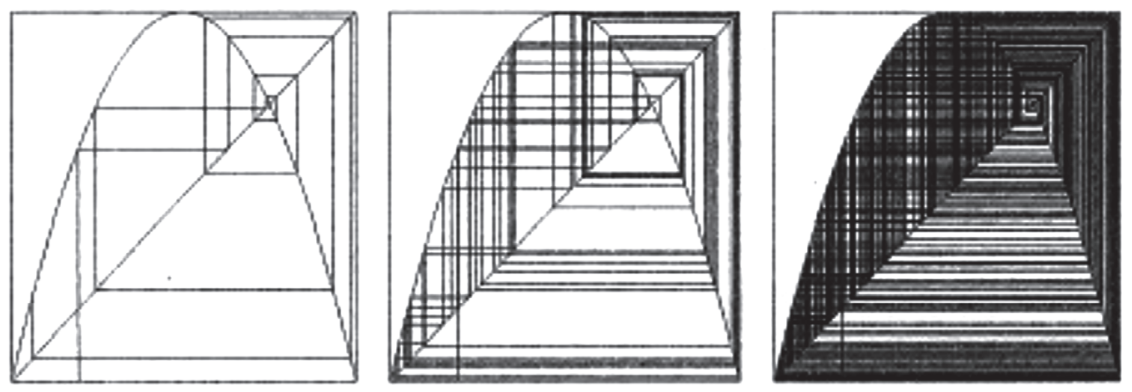

Fuente: Martín et al. (1998).

Si bien los sistemas dinámicos no lineales son ecuaciones deterministas, en tanto se definen a partir de una regla precisa de comportamiento, en el largo plazo pueden tener un alto componente de impredecibilidad. Aunque se escojan condiciones iniciales muy similares, una misma regla, un mismo sistema dinámico, presentará una evolución caótica de sus órbitas para ciertos parámetros. El comportamiento caótico y determinista que pueden presentar los sistemas dinámicos no lineales es de este modo un desafío a las distinciones señaladas al principio de este artículo. Azar y necesidad son categorías que adquieren un nuevo significado desde la no linealidad y temporalidad propia de este tipo de sistemas complejos. 


\section{INVESTIGANDO CON MODELOS DINÁMICOS NO LINEALES}

Ya con un contexto básico sobre las definiciones de sistemas dinámicos, es posible comprender más fácilmente las aplicaciones que se han desarrollado en ciencias sociales, así como su nivel de profundidad y rigurosidad en relación a la teoría matemática que subyace a los conceptos de no linealidad y temporalidad. Al revisar la bibliografía en que se presentan aplicaciones se encuentran diversos tipos de aproximaciones.

Si bien las aplicaciones tienen distintos niveles de profundidad o formalización, por lo general tienen en común los conceptos desarrollados anteriormente. En el ámbito de los sistemas electorales, se encuentra, por ejemplo, "From Individuals to Groups: The Aggregation of Votes and Chaotic Dynamics" (Richards, 1997), así como "Hopf Bifurcation in multiparty political systems with time delay in switching" (Khan, 2000). En el primero, específicamente, se estudia el comportamiento electoral entendido como agregación de preferencias individuales, las cuales tendrían un comportamiento no lineal en tanto incrementos en una variable no generan incrementos uniformes en la otra. El proceso mediante el cual se consigue un acuerdo social entre un grupo de individuos, cada uno con sus preferencias y habilidades para actuar estratégicamente, no se organiza de acuerdo a una simple relación aditiva donde el interés grupal es una mera suma de intereses individuales. También se han desarrollado modelos sobre conflicto bélico, destacándose los modelos de batalla propuestos por Lanchester, tales como "A verification of Lanchester's Law" (Engel, 1954), en que se proponen ecuaciones diferenciales para observar la dinámica entre las tropas enemigas. Otro terreno explorado en términos de sistemas dinámicos corresponde a la dinámica epidemiológica, en que se estudia la difusión de enfermedades transmisibles o infecciosas en distinto tipo de poblaciones. Ciertamente existen una serie de aplicaciones, que pueden ser revisadas, entre otros, en el Journal of Mathematical Sociology.

Nótese que el objetivo de este artículo es más introducir a una lógica de investigación sobre la modelación de cierto tipo de sistemas dinámicos en ciencias sociales, que describir un panorama exhaustivo sobre los tipos de aplicaciones existentes. En este sentido, más allá de realizar una descripción detallada de cada una de las experiencias de modelación con sistemas dinámicos en ciencias sociales, se opta por profundizar en dos cuestiones clave relevantes en este campo: en primer lugar, una explicación detallada de los modelos epidemiológicos, su lógica y pertinencia en temas de salud pública, así como problemas sociales que pueden tener una formalización similar. En segundo lugar, la presentación de una aplicación completa de un sistema dinámico en el ámbito de la economía, ocupándose de cuestiones de orden metodológico y tratando de cubrir los principales elementos en una investigación en ciencias sociales.

\subsection{Modelos epidemiológicos}

Un conjunto importante de aplicaciones de sistemas dinámicos en ciencias sociales se desarrollan a partir de lo que se conoce como las matemáticas de las enfermedades infecciosas (Hethcote, 2000). Éstas consisten en el estudio de la dinámica epidemiológica, 
enfatizando la modelación de enfermedades que por su carácter transmisible presentan un comportamiento en que la predicción resulta compleja. De este modo, este tipo de modelos resulta interesante para las ciencias sociales en al menos dos sentidos.

En primer lugar, en términos de la importancia para la salud pública que el control de las enfermedades infecciosas tiene a nivel mundial, y en especial para los países de América Latina y el Caribe, en que si bien han ido progresivamente dando paso a una mayor preponderancia de enfermedades crónicas, aún se presenta una alta coexistencia con cierto tipo de enfermedades transmisibles (CEPAL, 2010). Esto adquiere relevancia en el contexto de la investigación sobre las determinantes sociales de la salud, en que se estudia, por ejemplo, la distribución diferenciada del riesgo de contagio según indicadores como nivel socioeconómico, etnia, segregación urbana, entre otros. En segundo lugar, en tanto siguiendo la lógica de la dinámica epidemiológica, es posible realizar ejercicios de modelación en otros ámbitos de las ciencias sociales, tal y como se demuestra en Joshua Epstein (1997) en el estudio de la difusión de drogadictos en una comunidad.

En Hethcote (2000) se describen los principios para formalizar la difusión del contagio de enfermedades transmisibles en un modelo particular ${ }^{5}$. Por lo general, el proceso de contagio se estudia a partir de etapas etiquetadas con las letras M, S, E, I y R, tal y como se ejemplifica en la Figura 4. Así, para una enfermedad que se presenta en niños y niñas recién nacidos, la etapa $M$ corresponde al grupo que presenta inmunidad por haber recibido anticuerpos a través de la placenta de madres que habían sido infectadas previamente. Luego que la inmunidad desaparece, este grupo se une al resto de niños en la etapa S, lo cual indica que son susceptibles de contagiarse. Aquellos casos que hayan tenido un contacto con población infectada, pasarán a formar parte del grupo E, por lo que mantienen la enfermedad en un estado latente sin capacidad de contagiar a más personas. Después de esta etapa, los niños pasan al grupo I, en que pueden transmitir la enfermedad a otros. Cuando I termina, el grupo pasa a la etapa $R$, en que además de estar recuperados, adquieren inmunidad. Es importante destacar que este esquema será diferente para cada tipo de enfermedad, por lo que es decisión del equipo que desarrolla el modelo el ordenamiento a seguir, así como las etapas que se incluirán en la formalización.

La incidencia de la enfermedad por contagio horizontal que se muestra en la Figura 4 expresa la infección de la población susceptible mediante el contacto con la población infectada. En este sentido, si $S(t)$ es el número de personas susceptibles en el tiempo $t, I(t)$ es el número de infectados, y $N$ es el tamaño de la población total, entonces $s(t)=S(t) / N$ y $i(t)=I(t) / N$ son las fracciones de población susceptible e infectada, respectivamente. Si $\beta$ es el número promedio de contactos adecuados (contactos suficientes para la transmisión de la enfermedad) de una persona por unidad de tiempo, entonces $\beta I / N=\beta i$ es el número promedio de contactos con infectados por unidad de tiempo de un susceptible, y $(\beta I / N)$ $S=\beta$ Nis es el número promedio de nuevos casos por unidad de tiempo, ya que $S=N s$. Es clave destacar que el cálculo del valor $\beta$ dependerá de los patrones específicos de contacto

5 Para una revisión de un conjunto más amplio de modelos epidemiológicos, véase Hethcote (1994). 
FIGURA 4

Diagrama de transmisión general para modelos MSEIR

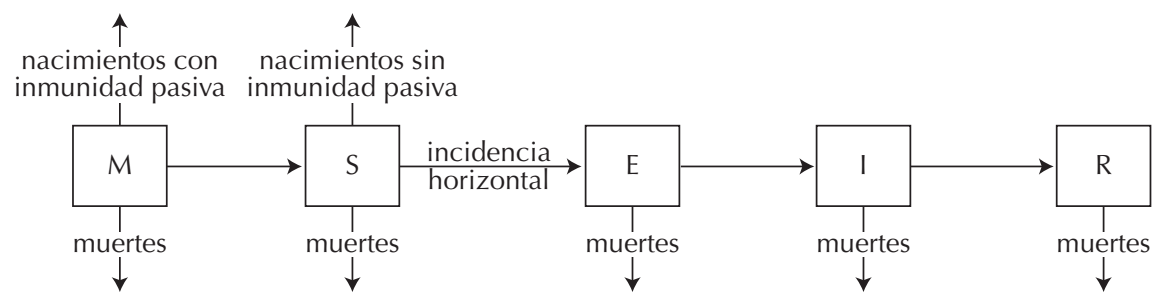

Fuente: Sobre la base de Hethcote (2000).

entre los individuos de la población en que se presente la epidemia, por lo que no corresponde asumir una proporcionalidad entre este parámetro y el tamaño de la población.

Uno de los aspectos clave de la dinámica de las enfermedades infecciosas está dado por los parámetros conocidos como la tasa de reproducción básica $R o$, tasa de contacto $\sigma$ y la tasa de reemplazo $R$, en tanto definen los umbrales que determinan que una enfermedad sea capaz de invadir una población en particular. Ro corresponde al número de casos secundarios de contagio provenientes de un infectado. Así, si $R o>1$ se tiene equilibrio endémico y si $R o<1$ se tiene un caso de control de la epidemia. En segundo lugar, $\sigma$ está definido por el número promedio de contactos adecuados que un infectado puede realizar durante un periodo infeccioso ${ }^{6}$. El número de reemplazo, $R$, se define como el número promedio de infecciones producidas en cada nuevo periodo infeccioso. Nótese que la relación entre estos tres parámetros es la siguiente: $R O \geq \sigma \geq R$, lo cual se explica porque en la mayor parte de las enfermedades $\sigma$ es igual a Ro. Además, el valor de $R$ va disminuyendo a medida que la prevalencia de la infección aumenta.

De este modo, uno de los modelos clásicos de la dinámica epidemiológica está dado por:

$$
\begin{array}{ll}
\frac{d S}{d t}=-\beta I S / N, & S(0)=S_{0} \geq 0 \\
\frac{d l}{d t}=-\beta I S / N-\gamma I, & I(0) \geq 0 \\
\frac{d R}{d t}=\gamma I, & R(0)=R_{0} \geq 0
\end{array}
$$

donde $S(t)+I(t)+R(t)=N$. Es conveniente enfatizar que si

$$
S(0)+I(0)+R(0)=N,
$$

6 El número promedio de contagios que podría generar si todos sus contactos fueran con individuos susceptibles. 


\section{CUADRO 1}

Términos para modelos basados en MSEIR

\begin{tabular}{|l|l|}
\hline \multicolumn{1}{|c|}{ VARIABLE } & \\
\hline $\mathrm{M}$ & Niños con inmunidad pasiva. \\
\hline $\mathrm{S}$ & Población susceptible. \\
\hline $\mathrm{E}$ & Personas expuestas en el periodo de infección latente. \\
\hline $\mathrm{I}$ & Infectados. \\
\hline $\mathrm{R}$ & Personas recuperadas y que, por lo tanto, desarrollan inmunidad. \\
\hline $\mathrm{m}, \mathrm{s}, \mathrm{e}, \mathrm{i}, \mathrm{r}$ & Fracciones de la población. \\
\hline$\beta$ & Tasa de contacto. \\
\hline $1 / \delta$ & Periodo de inmunidad pasiva promedio. \\
\hline $1 / \varepsilon$ & Periodo de infección latente promedio. \\
\hline $1 / \gamma$ & Periodo infeccioso promedio. \\
\hline Ro & Número (tasa) de reproducción básico. \\
\hline$\sigma$ & Número (tasa) de contacto adecuados. \\
\hline $\mathrm{R}$ & Tasa de reemplazo. \\
\hline
\end{tabular}

entonces de (1) se deduce que

$$
\frac{d S}{d t}(t)+-\frac{d l}{d t}(t)+\frac{d R}{d t}=0
$$

por lo que $S(t)+I(t)+R(t)$ es constante e igual a $N$ para todo $t$.

Nótese que este modelo SIR corresponde a un caso particular del modelo MSEIR presentado en el diagrama de la Figura 4.

Este modelo SIR utiliza un tasa de recuperación de $\gamma l$, lo que corresponde a un tiempo exponencial de espera $e^{-\gamma t}$. Al dividir las ecuaciones de 1 por el valor de la población total $N$ se obtiene:

$$
\begin{array}{ll}
d s / d t=-\beta i s, & \text { s }(0)=s_{0} \geq 0 \\
d i / d t=\beta i s-\gamma i, & \text { i }(0)=i_{0} \geq 0
\end{array}
$$


$\operatorname{con} r(t)=1-s(t)-i(t)$

Una mirada al espacio de fases definido como $T$, para las variables de $(s, i)$ en la Figura 5 , permite observar las soluciones de distintos valores en las condiciones iniciales de $s(0)$.

$$
T=\{(s, i) \mid s \geq 0, i \geq 0, s+i \leq 1\}
$$

FIGURA 5

Espacio de fases para modelo SIR

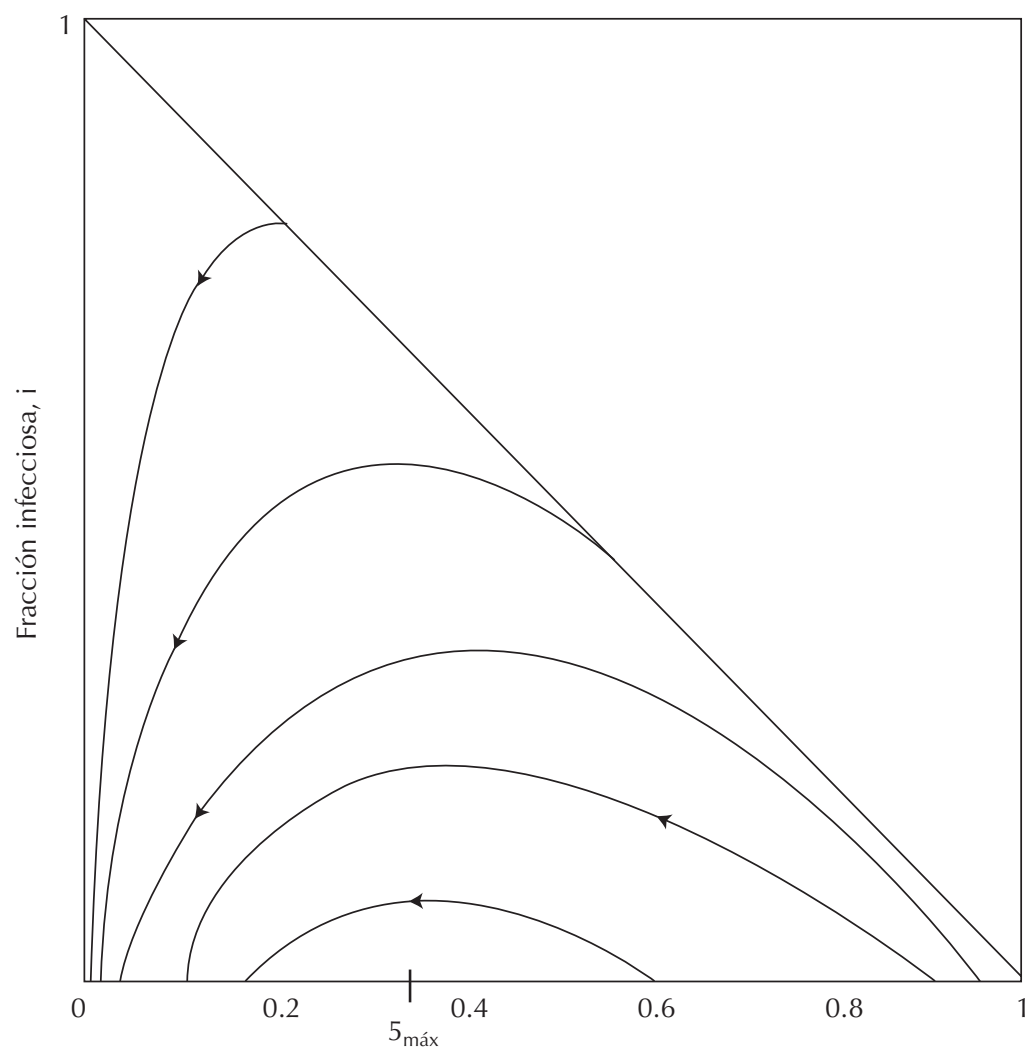

Fracción susceptible, s

Fuente: Sobre la base de Hethcote (2000).

Las soluciones del modelo para valores de $\sigma=3$ se presentan en la Figura 6. Así, se aprecia que la trayectoria de la población infectada es creciente hasta un punto máximo, para finalmente decrecer hasta 0 . Por otro lado, la población susceptible disminuye a medida que se 
FIGURA 6

Soluciones del modelo SIR

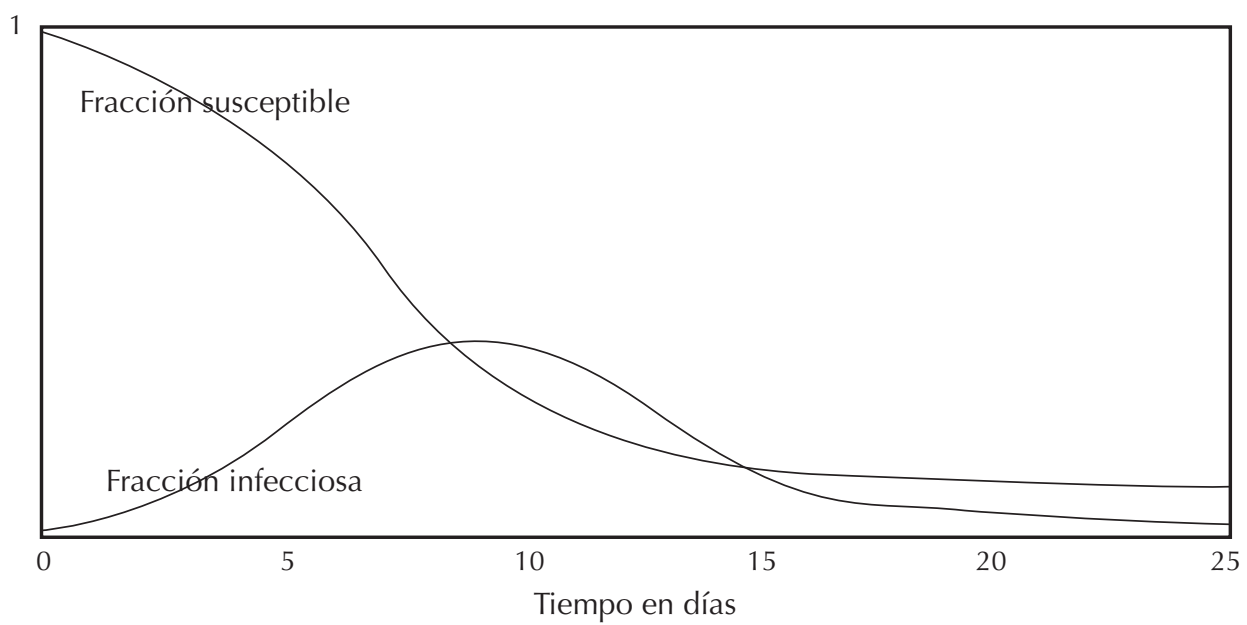

Fuente: Sobre la base de Hethcote (2000).

va convirtiendo en población infectada o recuperada, para luego alcanzar valores constantes. Aquí el número adecuado $\sigma=\beta / \gamma$ corresponde a la tasa de contacto $\beta$ por unidad de tiempo, multiplicado por el periodo de infección promedio $1 / \gamma$. Es importante destacar que en otros modelos epidemiológicos se han encontrado atractores caóticos (Diallo y Koné, 2007).

Por otro lado, Joshua Epstein, investigador del Santa Fe Institute, es uno de los autores que ha realizado aplicaciones de sistemas dinámicos a ciencias sociales que resultan paradigmáticas en cuanto a su vinculación con las matemáticas de las enfermedades infecciosas. De este modo, en Nonlinear Dynamics, Mathematical Biology and Social Science (Epstein, 1997) se destacan dos modelos, uno que trata de predecir la dinámica de la carrera armamentista entre dos países, y otro que explica la dinámica de la difusión de drogas en una comunidad.

En ambas aplicaciones se presentan preguntas similares a las que se realizan cuando se utiliza la ecuación logística para modelar el comportamiento de poblaciones biológicas o de difusión de epidemias. La motivación que subyace a estas aplicaciones es por un lado la idea de que existen modelos matemáticos simples que pueden iluminar dinámicas complejas. Así como el interés por profundizar formalmente en analogías entre problemas biológicos y sociales, en tanto la biología matemática ofrece un potencial subexplotado para desarrollar un nuevo punto de vista de la dinámica social. Es decir, si para preguntas sobre el crecimiento poblacional de insectos se responde a cuestiones como: ¿cuál será la variación de la cantidad de insectos entre la semana 5 y 6?; ¿en qué medida el crecimiento de la población de insectos depende de la densidad de ésta?; ¿ es siempre posible predecir la dinámica de la población o solo para ciertas tasas de reproducción?; ¿en qué condiciones se genera comportamiento caótico del crecimiento poblacional? Las aplicaciones desarrolladas 
por Epstein responden a interrogantes del tipo: ¿es posible que la carrera armamentista entre dos países se estabilice para cierta cantidad de armamento total, generando un ritmo de crecimiento predecible?; ¿ en qué situaciones no es posible predecir la dinámica de la carrera armamentista?; ¿cuál será la variación de la población de drogadictos de un año a otro?; ¿en qué sentido depende de la cantidad de drogadictos existentes en un momento determinado?

Estudiar la evolución del crecimiento de un grupo de drogadictos en una comunidad en que se interactúa con grupos de no adictos, policía y nuevos integrantes en la comunidad, es ciertamente un problema complejo, en tanto la dinámica de la expansión se basa en una relación no lineal entre los diferentes actores. Por un lado está el componente nolineal y por otro el de temporalidad, de ahí que se considere el uso de sistemas dinámicos para modelar el problema.

Si bien aquí no interesa profundizar en la formalidad matemática del modelo, es importante especificar cuáles son los elementos que Epstein toma en cuenta para describir la dinámica de la población de drogadictos en una comunidad. De este modo, es posible distinguir en el modelo cuatro grupos que tendrán una dinámica particular pero que al interactuar se modifican entre sí, así como la dinámica total de la comunidad.

Cada una de las ecuaciones diferenciales describe la dinámica de las variables presentadas, obteniendo un modelo de la dinámica total de la comunidad. Es interesante destacar que la complejidad del problema (modelar la dinámica de la expansión de drogadictos) puede ser estudiada con un sistema dinámico simple, muy similar al modelo SIR presentado anteriormente.

\section{CUADRO 2}

Términos del modelo

\begin{tabular}{|c|l|}
\hline VARIABLE & \multicolumn{1}{|c|}{ DEFINICIÓN } \\
\hline $\mathrm{S}(\mathrm{t})$ & Población no adicta y susceptible. \\
\hline $\mathrm{I}(\mathrm{t})$ & Población adicta. \\
\hline $\mathrm{L}(\mathrm{t})$ & Policía. Su función consiste arrestar a los adictos de la comunidad. \\
\hline $\mathrm{R}(\mathrm{t})$ & Población arrestada. Se asume como permanente. \\
\hline$B$ & Tasa en la cual el contacto entre un adicto y no adicto produce un nuevo adicto. \\
\hline$M$ & Tasa de incorporación de nuevos integrantes a la comunidad. \\
\hline$\Gamma$ & Tasa en la cual el contacto entre policías y adictos resulta en el arresto de un adicto. \\
\hline$\alpha$ & Tasa en la cual el incremento de adictos resulta en incremento de policía. \\
\hline $\mathrm{b}$ & Amortiguamiento económico al cual está sujeto el crecimiento policial. \\
\hline
\end{tabular}


Lo cual se explica en tanto la no linealidad del problema se presenta básicamente en la interacción no lineal entre la población drogadicta y no drogadicta.

\subsection{Ejemplo de aplicación}

Una aplicación de sistemas dinámicos a ciencias sociales puede desarrollarse en los siguientes pasos. En primer lugar, es necesario identificar un problema de investigación en que el componente no lineal y temporal sea fundamental. En segundo lugar, se requiere de la selección, modificación o construcción de un modelo basado en sistemas dinámicos que consiga responder al problema de investigación y de esta forma a los objetivos que se plantean en la investigación. En tercer lugar, se requiere realizar simulaciones computacionales que permitan observar los distintos escenarios posibles a partir de situaciones iniciales específicas. Finalmente se requiere discutir los resultados y plantear conclusiones a partir de éstos. De este modo, el siguiente ejemplo de aplicación permite mostrar la lógica de una aplicación en que el uso de sistemas dinámicos sea relevante. El problema se enmarca en el ámbito de la pesca artesanal, en tanto puede ser un tema complejo si se toma en cuenta la relación entre los aspectos biológicos y económicos.

\subsubsection{Presentación del problema y justificación del componente no lineal y temporal}

La sostenibilidad de la pesca artesanal entendida como un problema complejo presenta relaciones no lineales entre los aspectos biológicos y económicos. En términos intuitivos significa, por ejemplo, que mayores niveles de esfuerzo pesquero (cantidad de embarcaciones, principalmente) de los recursos marinos no tienen necesariamente mayores beneficios económicos ni mayor impacto biológico. De hecho, en algunos casos conviene explotar más el recurso para que éste se reproduzca más rápido, pero en otras se requiere hacer precisamente lo contrario. Por otro lado, las decisiones sobre sostenibilidad no pueden ser de carácter estático, ya que cuestiones que son válidas en un momento, no necesariamente lo son en otro.

En particular, en la pesquería de merluza común de la quinta región de Chile no hay claridad sobre la situación futura de los pescadores artesanales, en cuanto a si en el mediano y largo plazo seguirá habiendo disponibilidad de peces dada la situación actual de esfuerzo pesquero, lo que tiene un directo impacto en los beneficios económicos, así como en el ingreso de nuevos pescadores a la pesquería. En este sentido, la pregunta que motiva la presente aplicación sería: ¿Es sostenible en términos biológicos y económicos la pesca artesanal de la quinta región en Chile a partir de su situación actual?

\subsubsection{Selección del modelo}

Si bien no interesa caer en tecnicismos matemáticos, ya que el trabajo de aplicaciones de sistemas dinámicos se desarrolla con equipos interdisciplinarios en que cada investigador aporta desde su propio campo, hay que tener presentes algunas nociones básicas del proceso de modelación para facilitar la comunicación (Carrasco, 2008). La comprensión del planteamiento 
del problema y así como se verá más adelante, las simulaciones computacionales, requieren al menos un manejo intuitivo del funcionamiento del proceso de modelación matemática.

De los diversos tipos de sistemas dinámicos existentes para el estudio de sostenibilidad pesquero, se opta por seleccionar el modelo bioeconómico de Gordon-Schaefer, en tanto si bien es uno de los más simples, permite acercarse a responder a la pregunta de investigación. La justificación para la selección de este modelo es básicamente que la pregunta por la sostenibilidad en términos biológicos y económicos de un recurso pesquero requiere de un modelo que "relacione la reproducción de un recurso renovable, con su explotación motivada por fines económicos" (Franquesa, 1995). Específicamente, este modelo tiene que ser capaz de relacionar la tasa de reproducción de un recurso vivo, con el esfuerzo pesquero como un costo, así como las capturas o cantidades de pesca expresadas como un ingreso por las ventas del recurso. Formalmente, el modelo de Gordon-Schaefer es el siguiente:

$$
\begin{gathered}
B_{t+1}=B_{t}+r B_{t}\left(1-\frac{1}{K} B_{t}\right)-C_{t} \\
\pi=I T S-C T=p C-c E
\end{gathered}
$$

Donde los términos del modelo son:

\section{CUADRO 3}

Términos del modelo Gordon-Schaefer a tiempo discreto

\begin{tabular}{|l|l|}
\hline$B_{t+1}$ & Dinámica de la población. \\
\hline$C_{t}=q \mathrm{E} B_{t}$ & Captura E: Esfuerzo de pesca. \\
\hline$K$ & Capacidad de carga del ecosistema. \\
\hline$r$ & Tasa de reproducción intrínseca. \\
\hline$q$ & Coeficiente de capturabilidad. \\
\hline$C$ & Costo. \\
\hline$p$ & Precio. \\
\hline$t$ & Tiempo. \\
\hline$\pi$ & Beneficios económicos. \\
\hline$I T S$ & Ingresos totales. \\
\hline$C T$ & Costos totales. \\
\hline
\end{tabular}


Básicamente, es importante tener claro dos elementos. En primer lugar, que el modelo se construye a partir de la ya mencionada ecuación logística. Por lo que por un lado se está modelando el crecimiento poblacional de peces y las capturas (cantidades de pesca realizada) que se realizan producto de la explotación pesquera. Por otro lado, en la segunda ecuación se modelan los beneficios económicos, relacionando ingresos y costos, con capturas y esfuerzo pesquero, respectivamente.

En segundo lugar, que la relación entre el aspecto biológico y económico es no lineal en tanto las capturas dependerán a su vez de la cantidad de biomasa (cantidad de peces en el mar) como de esfuerzo pesquero existente. De este modo, lo que se plantea en el modelo es que las capturas y por lo tanto los beneficios económicos no tienen, a medida que pasa el tiempo, una relación lineal con las cantidades de biomasa.

\subsubsection{Simulaciones}

A continuación se presentan las salidas computacionales luego de haber realizado las simulaciones con datos inventados. El programa computacional usado es el paquete informático Matlab, para lo cual se definieron los algoritmos necesarios para expresar el comportamiento del modelo en términos de la sintaxis particular. De manera similar, es posible utilizar el programa Scilab, que cuenta con una plataforma de fuente abierta y descarga gratuita del sitio web.

La primera y la segunda salidas representan diferentes posibilidades de la pesquería, permitiendo observar los puntos de equilibrio entre el aspecto biológico y económico. La tercera y la cuarta salidas computacionales muestran las simulaciones propiamente tales, prediciendo la evolución de la cantidad de biomasa y de los beneficios económicos a partir de la cantidad actual de esfuerzo pesquero del caso estudiado. Se utilizan los datos del Cuadro 4.

En la Figura 7 se muestra cómo la situación de esfuerzo pesquero genera una cantidad de capturas que no es sostenible en el tiempo, de ahí que el modelo prediga que las capturas se estabilizarán en algún punto a lo largo de la curva de "Ingresos sostenibles". En la Figura 8 se puede observar la relación biológica y económica de la pesquería en cuestión, en tanto hay incentivos económicos para que el esfuerzo pesquero aumente hasta 7.000 embarcaciones aproximadamente. Sin embargo, esa situación sería una disminución dramática en términos de biomasa.

En la Figura 9 se observa que la cantidad de beneficios se estabiliza en una cantidad compatible con el nivel de esfuerzo, lo cual da cuenta tanto de la disminución de las capturas como de biomasa. De este modo, dada la condición inicial actual, las soluciones se estabilizan en los diferentes puntos de equilibrio (Clark, 1990). Estos puntos de equilibrio están representados a lo largo de toda la curva de la Figura 7 y en términos de la teoría de sistemas dinámicos, se definen como puntos atractores ya que "atraen" las soluciones o trayectorias hacia ellos. En este caso, considerando que el esfuerzo pesquero definido es de 300 embarcaciones, el punto atractor que le corresponde a las trayectorias se obtiene 


\section{CUADRO 4}

Datos utilizados para la realización de las simulaciones

\begin{tabular}{|l|l|l|}
\hline \multicolumn{1}{|c|}{ NOTACIÓN } & \multicolumn{1}{|c|}{ VALOR } & \multicolumn{1}{c|}{ UNIDAD DE MEDIDA } \\
\hline K: capacidad de carga del ecosistema & 600.000 & Toneladas \\
\hline r: tasa de reproducción intrínseca & 0.3 & - \\
\hline q: coeficiente de capturabilidad & 0.00004 & - \\
\hline B0: biomasa inicial & 600.000 & Toneladas \\
\hline E: esfuerzo realizado por flota artesanal & 300 & Embarcaciones \\
\hline c: costo & 15.000 & Pesos chilenos por día \\
\hline p: precio & 246.000 & Pesos por tonelada \\
\hline t: tiempo & $1: 20$ & Años \\
\hline
\end{tabular}

FIGURA 7

Captura sostenible versus esfuerzo artesanal

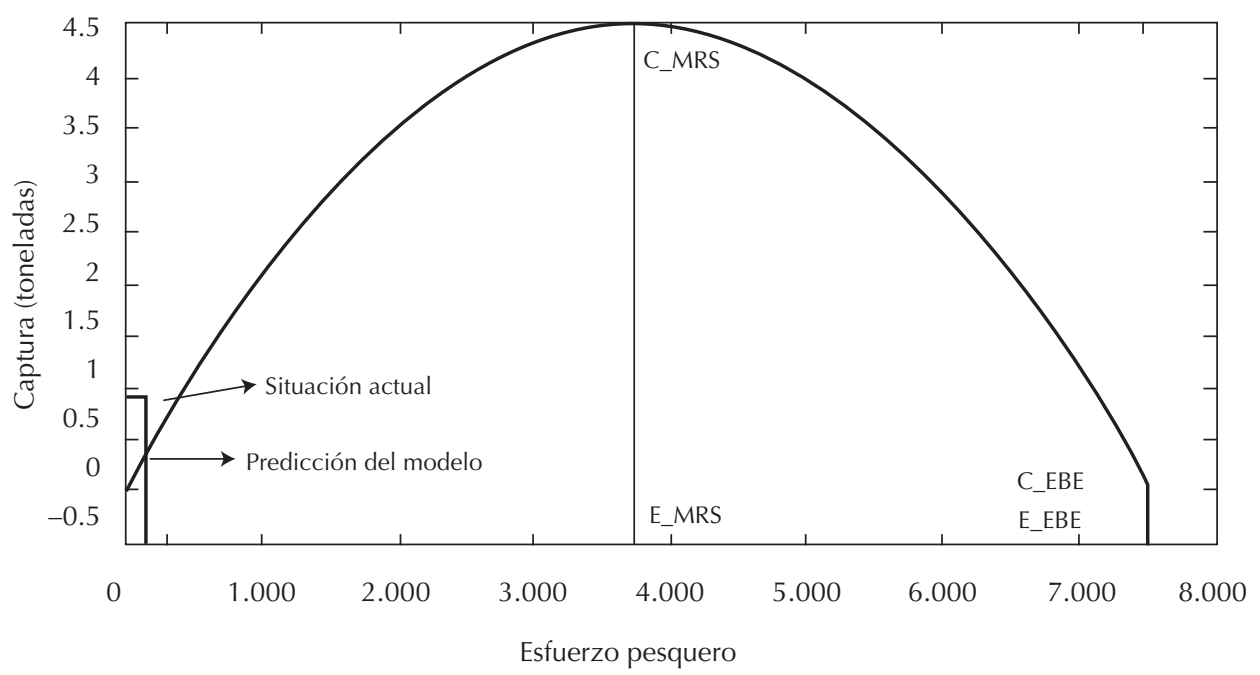

Fuente: Salidas computacionales Matlab. 
FIGURA 8

Ingresos y costos sostenibles
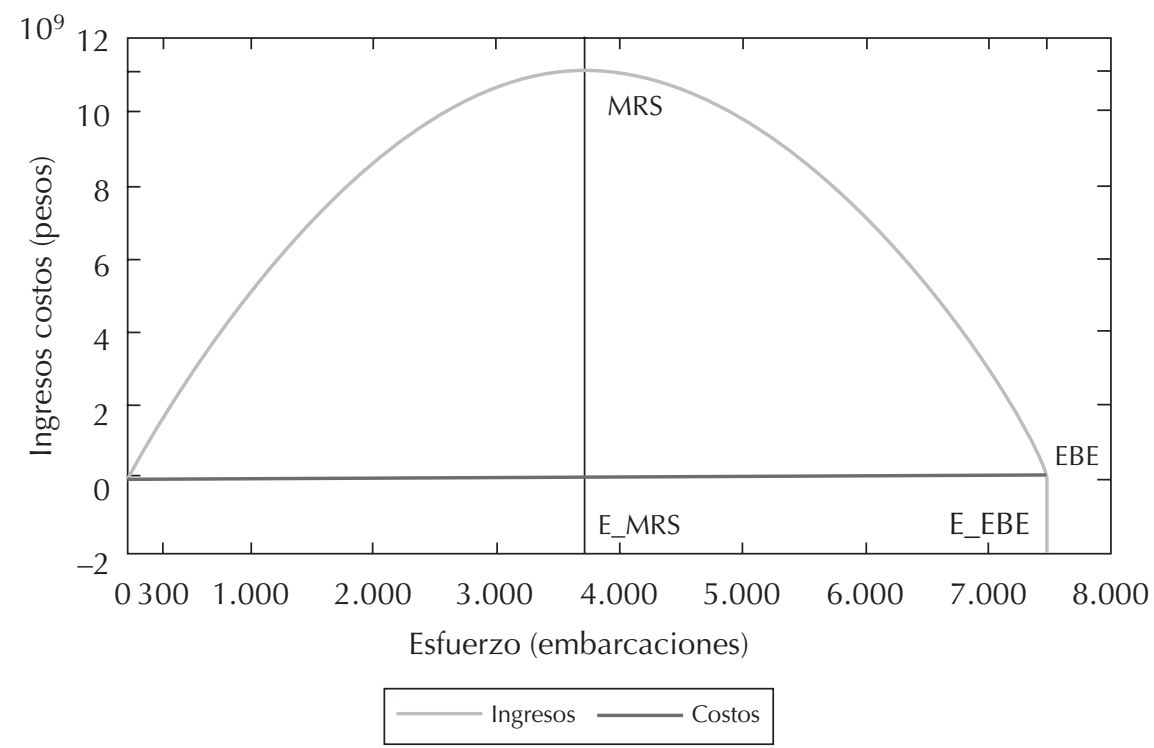

Fuente: Salidas computacionales Matlab.

FIGURA 9

Simulación evolución de captura

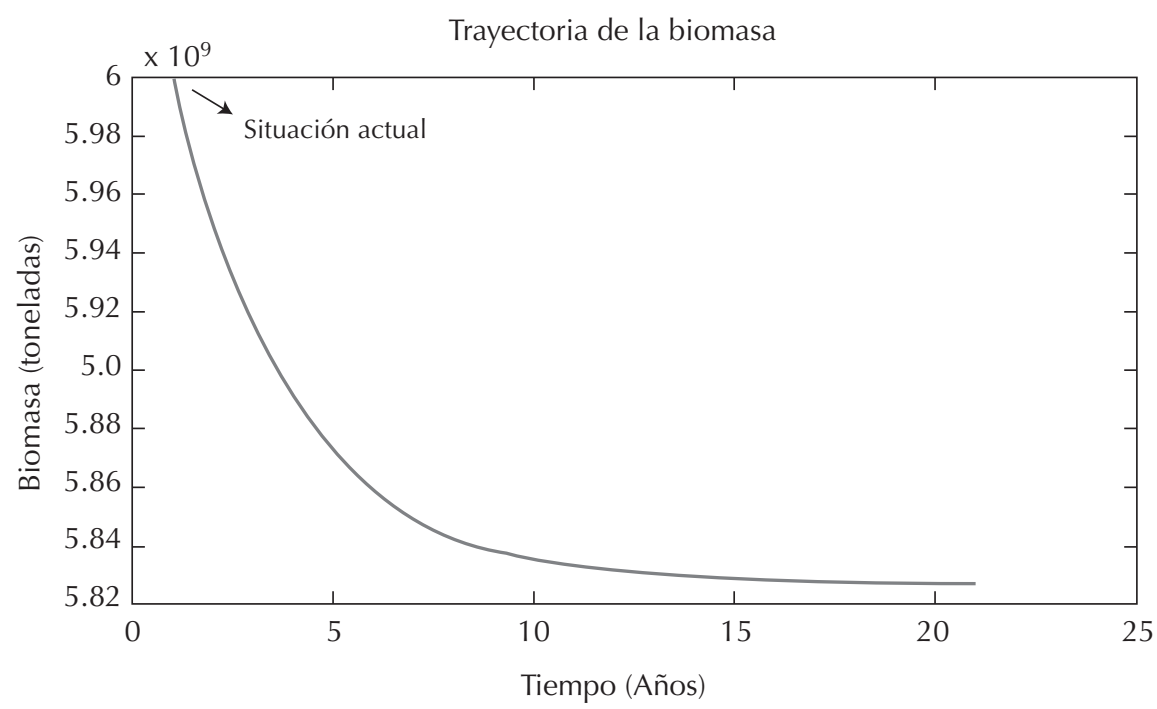

Fuente: Salidas computacionales Matlab. 
proyectando una línea recta desde esa cantidad de esfuerzo hacia la curva de biomasa representada en la Figura 7.

Considerando las limitaciones que el presente modelo tiene, principalmente en cuanto no considera esfuerzo pesquero industrial y por lo tanto su validez se ve muy reducida ${ }^{7}$, es posible señalar algunas interpretaciones a partir de las simulaciones realizadas. La situación de la pesca artesanal de la merluza común en Valparaíso sería sostenible en términos biológicos y económicos en caso de que sólo se considerara este tipo de pesca. Sería sostenible en términos biológicos, en tanto si bien la cantidad de capturas son superiores a las sostenibles, en el plazo simulado éstas disminuirían hasta alcanzar una cantidad compatible para que la biomasa se mantenga constante. Económicamente, ya que si bien los beneficios económicos de los pescadores artesanales disminuirían producto de la disminución de las capturas, también se estabilizarían en un nivel en que, según el modelo, sigue siendo rentable la cantidad de esfuerzo pesquero.

\section{DISCUSIÓN}

Sobre la base de las definiciones, distinciones y aplicaciones de sistemas dinámicos en ciencias sociales presentadas, surgen a lo menos dos interrogantes que vale la pena seguir investigando. En primer lugar, reflexionar sobre el sentido de obtener cierto tipo de resultados. Muchas de las aplicaciones de sistemas dinámicos se esfuerzan por encontrar atractores extraños o comportamiento caótico en series de datos, pero el desempeño explicativo que esto tiene no se profundiza mayormente. Por ejemplo, ¿qué significa en términos sociológicos que el comportamiento de un modelo en particular sea caótico para ciertos parámetros? Ciertamente cuando se obtienen correlaciones entre dos o más variables, para cualquier cientista social es posible hacer una interpretación, incluso si no se conoce el trasfondo matemático de la técnica de análisis.

Finalmente, es clave reflexionar sobre la pertinencia de desarrollar modelación matemática en ciencias sociales actualmente. Los sistemas dinámicos, y en especial las ecuaciones diferenciales ordinarias presentadas en este artículo, son parte de un conjunto más amplio de posibilidades para abordar teórica y empíricamente la complejidad de la sociedad. En este sentido, considerando que se trata de herramientas de análisis y de investigación que aún se encuentran en un estado incipiente, se requiere tener claridad sobre el contexto en que pueden ser útiles: no linealidad y temporalidad.

\section{COMUNIDAD Y HERRAMIENTAS}

- Center for Chaos and Complexity.

- Chaos for Java, XPPAUT, Phaser.

- Discrete and Continuous Dynamical Systems.

- Dynamical System. An International Journal.

7 Para una revisión de un artículo en que se considera el esfuerzo pesquero industrial, véase Martinet et al. (2010). 
- Dynamics of Continuous, Discrete and Impulsive Systems.

- Journal of Biological Dynamics.

- Journal of Difference Equations and Applications.

- Matlab tiene un módulo para estudiar dinámica no lineal.

- Max Planck Institute for Dynamics and Self-organization.

- SCILAB se especializa en modelos caóticos.

- Society for Chaos Theory in Psychology and Life Sciences.

- The Society for Non Linear Dynamics in Economy.

\section{BIBLIOGRAFÍA}

Berge, P., Yves Pomeau y Christian Vidal (1994): Order within Chaos, Wiley and Sons, Paris.

Berry, B. y Heja Kim (1997): “Long Waves 1790-1990: Intermittency, Chaos, and Control”, en D. Kiel y Euel Elliot (eds.): Chaos Theory in the Social Sciences. Foundations and Applications, University of Michigan Press, Ann Arbor.

Besicovitch, A.S. (1952): Almost Periodic Functions, Dover.

Capra, F. (1998): Las Conexiones ocultas, Anagrama, Barcelona.

Carrasco, J. Ignacio (2008): “Consideraciones metodológicas para la aplicación de sistemas dinámicos en ciencias sociales", Tesis para optar al título de sociólogo, Universidad de Chile, Santiago de Chile.

Castoriadis, C. (1993): La Nuit de Ville-Evrard. Temps, Memoires, Chaos, Descartes et Compagnie, Paris.

CEPAL (2010): Población y salud en América Latina y el Caribe: retos pendientes y nuevos desafíos, Naciones Unidas, Santiago de Chile.

Chatelet, G. (1995): Vivre et penser comme des porcs, Gallimard, Paris.

Clark, C. (1990): Mathematical Bioeconomics. The Optimal Management of the Renewable Resources. Wiley and Sons, New York.

Cohen, J. e lan Stewart (1995): The Collapse of Chaos, Penguin Books, London.

Devaney, R. (2003): An Introduction to Chaotical Dynamical System, Westview Press, Colorado.

Diallo, O. e Y. Koné (2007): "Melkinov analysis of chaos in a general epidemiological model", Nonlinear Anal. Real World Appl., 8, pp. 20-26.

Ditto, W. y L. Percora (1993): "Mastering Chaos", Scientific American, agosto, p. 62.

Engel, J.H. (1954): "A Verification of Lanchester's Law", Operations Research, 2, pp. 163-171.

Epstein, J. (1997): Non Linear Dynamics. Mathematical Biology and Social Science, Addison Wesley, Redwood City.

Franquesa, R. (1995): Modelos Bioeconómicos y políticas de regulación pesquera. Una lección introductoria, Gabinete de Economía del Mar, España.

Gleick, J. (1988): Caos: la Creación de una Nueva Ciencia, Seix Barral, Barcelona.

Grassberger, P. e I. Procaccia (1983): "Characterization of strange attractors", Physical Review Letters, 50, pp. 448-451. 
Hethcote, H. (1994): "A thousand and one epidemic models", en S. Levin (ed.): Frontiers in Mathematical Biology, Lecture Notes in Biomathematics, 100, Springer, Berlin, pp. 504-515.

(2000): "The Mathematics of infectious diseases", SIAM Review, 42, pp. 599-653.

Kauffman, S. (2003): Investigaciones, Tusquets, Barcelona.

Kelley, Walter y A. Peterson (2000): Difference equations: an introduction with applications, Academic Press, San Diego.

Khan, Q.J.A. (2000): "Hopf bifurcation in multiparty political systems with time delay in switching", Applied Mathematics Letters, 13 (7), pp. 43-52.

Kiel, D. y Euel Elliot (eds.) (1997): Chaos Theory in the Social Sciences. Foundations and Applications, University of Michigan Press, Ann Arbor.

Langton, C. (1990): "Chaos Computation at the Edge of Chaos", en S. Forrest (comp.): Emergent Computation, MIT Press, Cambridge.

Martín, M.A., M. Morán y M. Reyes (1998): Iniciación al Caos, Síntesis, Madrid.

Martinet, V., J. Peña, M. de Lara y H. Ramírez (2010): Evaluation of management procedures: Application to Chilean Jack Mackerel fishery, Proceeding of Economics of Fish Resources and Aquatic Ecosystems (IIEFT), Montpellier.

Murray, J. (1989): Mathematical Biology, Springer, New York.

Richards, D. (1997): "From Individual to Groups: The Aggregation of Votes and Chaotic Dynamics", en D. Kiel y Euel Elliot (eds.): Chaos Theory in the Social Sciences. Foundations and Applications, University of Michigan Press, Ann Arbor.

Ruelle, D. y Floris Takens (1971): "On the nature of the turbulence", Communication in Mathematical and Physics, 20 (3), pp. 167-192.

Saber, N. (1991): An introduction to difference equations, Springer, New York.

Thieme, H. (2003): Mathematical Population Biology, Princeton University Press, Princeton

Vivanco, M. (2010): Complejidad y sociedad: del discurso al modelo, LOM, Santiago de Chile.

Waldorp, M. (1992): Complexity: The Emerging Science at the Edge of Order and Chaos, Viking, London.

Recibido: 04-10-2010

Aceptado: 26-01-2011 